\title{
Defects-driven appearance of half-metallic ferrimagnetism in Co-Mn-based Heusler alloys
}

\author{
K. Özdog̃an ${ }^{\mathrm{a}}$ I. Galanakis ${ }^{\mathrm{b}, *}$ E. Şaşıog̃lu ${ }^{\mathrm{c}, \mathrm{d}}$ B. Aktaş ${ }^{\mathrm{a}}$ \\ ${ }^{a}$ Department of Physics, Gebze Institute of Technology, Gebze, 41400, Kocaeli, Turkey \\ b Department of Materials Science, School of Natural Sciences, University of Patras, GR-26504 Patra, Greece \\ ${ }^{\mathrm{c}}$ Institut für Festkörperforschung, Forschungszentrum Jülich, D-52425 Jülich, Germany \\ ${ }^{\mathrm{d}}$ Fatih University, Physics Department, 34500, Büyükçekmece, İstanbul, Turkey
}

\section{Abstract}

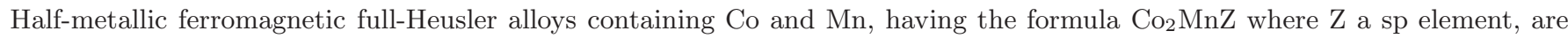
among the most studied Heusler alloys due to their stable ferromagnetism and the high Curie temperatures which they present. Using state-of-the-art electronic structure calculations we show that when Mn atoms migrate to sites occupied in the perfect alloys by Co, these $\mathrm{Mn}$ atoms have spin moments antiparallel to the other transition metal atoms. The ferrimagnetic compounds, which result from this procedure, keep the half-metallic character of the parent compounds and the large exchange-splitting of the $\mathrm{Mn}$ impurities atoms only marginally affects the width of the gap in the minority-spin band. The case of $\left[\mathrm{Co}_{1-x} \mathrm{Mn}_{x}\right]_{2} \mathrm{MnSi}$ is of particular interest since $\mathrm{Mn}_{3} \mathrm{Si}$ is known to crystallize in the Heusler $L 2_{1}$ lattice structure of $\mathrm{Co}_{2} \mathrm{MnZ}_{\mathrm{Z}}$ compounds. Robust half-metallic ferrimagnets are highly desirable for realistic applications since they lead to smaller energy losses due to the lower external magnetic fields created with respect to their ferromagnetic counterparts.

Key words: A.magnetically ordered materials

PACS: 75.47.Np, 75.50.Cc, 75.30.Et

\section{Introduction}

The emergence of spintronics, also known as magnetoelectronics, the last decade brought in the center of scientific research new properties and materials which had received little attention in the past [1]. An important class of materials which are at the present under intense study are the so-called half-metals [2]. These materials are hybrids between metals and semiconductors or insulators, presenting metallic behavior for one spin-band and semiconducting for the other, and thus overall they are either ferroor ferrimagnets with perfect spin-polarization at the Fermi level [3]. de Groot and his collaborators in a pioneering paper published in 1983 predicted the existence of halfmetallicity in the case of the intermetallic Heusler alloy $\mathrm{NiMnSb}$ [4]. Since then several half-metallic ferromagnetic

\footnotetext{
* Corresponding author. Phone +30-2610-969925, Fax +30-2610969368

Email addresses: kozdogan@gyte.edu.tr (K. Özdog̃an), galanakis@upatras.gr (I. Galanakis), e.sasioglu@fz-juelich.de (E. Şaşığlu).
}

materials have been initially predicted by theoretical abinitio calculations and after synthesized experimentally.

Half-metallic Heusler alloys are expected to play a key role in realistic applications due to their very high Curie temperatures and their structural similarity to the widely used binary semiconductors crystallizing in the zinc-blende structure [5]. Initially the research was focused on the socalled half- or semi-Heusler compounds like NiMnSb $[3,6]$ but lately the interest has been shifted to the so called full-Heusler compounds and mainly to the ones containing $\mathrm{Co}$, like $\mathrm{Co}_{2} \mathrm{MnAl}$ or $\mathrm{Co}_{2} \mathrm{MnGe}$, which were already known since early 70's [7]. In early 90's it was argued in two papers by a japanese group that they should be half-metallic ferromagnets [8] and first-principles calculations by Picozzi et al [9] and Galanakis et al [10] in 2002 confirmed their predictions. These papers acted as an inspiration to experimentalists who devote a constantly increasing number of publications to the study of their properties (see Refs. $[2,3,11,12,13,14,15,16,17,18,19,20]$ for references to some of these studies). Both the origin of ferromagnetism [21] and half-metallicity [10] in these compounds are well understood. 
Although the research on half-metallic ferromagnets is intense, the ideal case would be a half-metallic antiferromagnet, also known as fully-compensated ferrimagnet [22], since such a compound would not give rise to stray flux and thus would lead to smaller energy consumption in devices. In the absence of such an ideal compound, half-metallic ferrimagnetism is highly desirable since such compounds would yield lower total spin moments than their ferromagnetic counterparts. Some perfect Heusler compounds like FeMnSb [23] and $\mathrm{Mn}_{2} \mathrm{VAl}$ [24] are predicted to present half-metallicity in conjunction with ferrimagnetism. Recently other routes to half-metallic ferrimagnetism have been studied like the doping of diluted magnetic semiconductors [25], and the inclusion of defects in Cr pnictides [26] and $\mathrm{Co}_{2} \mathrm{CrAl}$ (or $\mathrm{Si}$ ) full-Heusler alloys [27].

In this communication we will study the appearance of defects-driven ferrimagnetism in the popular $\mathrm{Co}_{2} \mathrm{MnZ}$ compounds where $\mathrm{Z}$ stands for $\mathrm{Al}, \mathrm{Ga}, \mathrm{Si}, \mathrm{Ge}$ or $\mathrm{Sn}$. Most of the theoretical studies on these compounds either are devoted to the perfect compounds $[10,28,29]$ or concern disorder between the Mn and the sp atoms or doping of the Mn sites (see Refs. $[30,31,32]$ and references therein). When a surplus of Mn atoms is created occupying the sites corresponding to Co atoms at the perfect compounds, $\left[\mathrm{Co}_{1-x} \mathrm{Mn}_{x}\right]_{2} \mathrm{MnZ}$ alloys, these $\mathrm{Mn}$ impurity atoms are shown to be antiferromagnetically coupled to the other Co and Mn atoms at the perfect lattice sites. The resulting ferrimagnetic compounds keep the half-metallic character of the perfect parent alloys. Interestingly due to the very high exchange splitting presented by the Mn impurity atoms, the width of the gap is only marginally affected contrary to the $\mathrm{Co}_{2} \mathrm{CrAl}$ and $\mathrm{Co}_{2} \mathrm{CrSi}$ where the creation of $\mathrm{Cr}$ antisites almost vanished the gap [27]. Thus the defects-driven half-metallic ferrimagnetism presented in this communication is of particular interest for realistic applications. Special attention should be given to $\left[\mathrm{Co}_{1-x} \mathrm{Mn}_{x}\right]_{2} \mathrm{MnSi}$ alloys since $\mathrm{Mn}_{3} \mathrm{Si}$ is known experimentally to crystallize in the Heusler $L 2_{1}$ lattice structure of $\mathrm{Co}_{2} \mathrm{MnZ}$ compounds with two equivalent type of $\mathrm{Mn}$ atoms in the unit cell [33]. On the contrary $\mathrm{Mn}_{3} \mathrm{Ge}$ and $\mathrm{Mn}_{3} \mathrm{Sn}$ compounds crystallize in an hexagonal structure [34] while no information is available on the $\mathrm{Mn}_{3} \mathrm{Al}$ or $\mathrm{Mn}_{3} \mathrm{Ga}$ compounds. We should also note here that Picozzi and collaborators studied in Ref. [35] the case of a single Mn antisite using a supercell structure in the case of $\mathrm{Co}_{2} \mathrm{MnSi}$ and $\mathrm{Co}_{2} \mathrm{MnGe}$ compounds. They had actually found that the Mn impurity atoms has a spin moment antiparallel to the other transition metal atoms but they had not considered the case of extensive defects or studied in detail the observed behavior in their calculations.

We employed the full-potential nonorthogonal localorbital minimum-basis band structure scheme (FPLO) to perform the electronic structure calculations and the coherent potential approximation (CPA) to simulate the creation of the Mn antisites [36]. We used the scalar relativistic formulation and thus the spin-orbit coupling was not taken into account. The exchange-correlation potential was chosen in the local spin density approximation

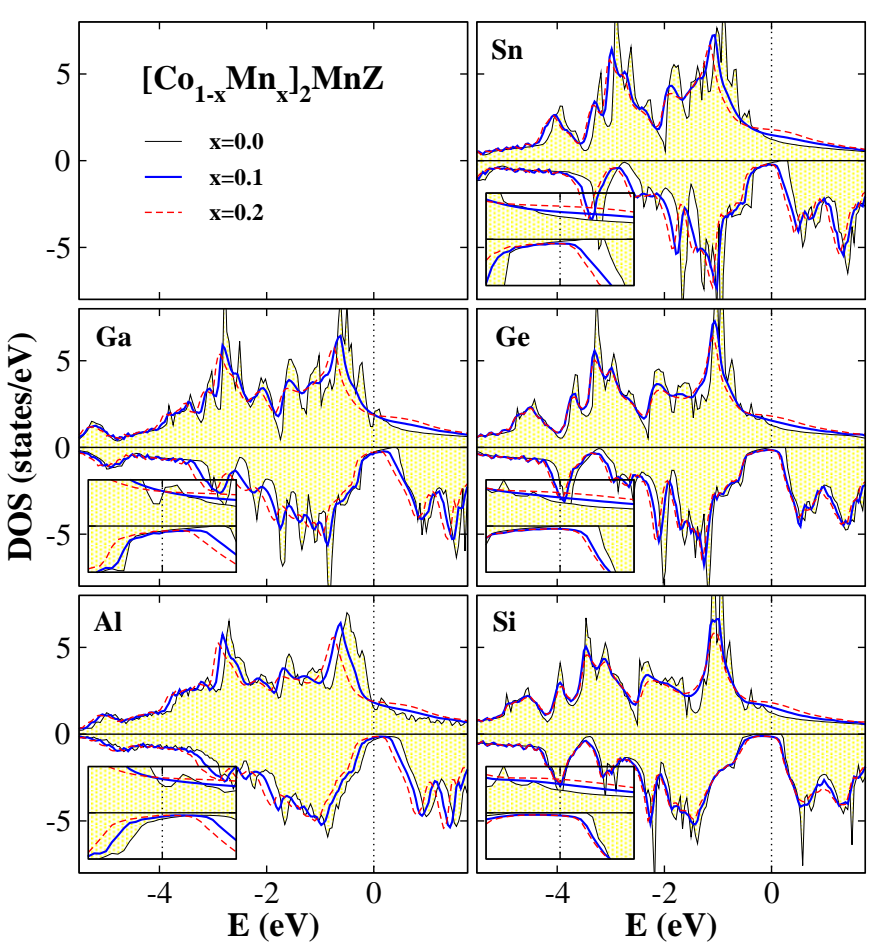

Fig. 1. Total density of states (DOS) for the $\left[\mathrm{Co}_{1-x} \mathrm{Mn}_{x}\right]_{2} \mathrm{MnZ}$ alloys as a function of the concentration $x$ : we denote $x=0$ with the solid grey line with shaded region, $x=0.1$ with a solid thick blue line and $x=0.2$ with a dashed red line. The Fermi level has been chosen as the zero of the energy axis, and positive values of DOS correspond to the spin-up (majority) electrons while negative values correspond to the spin-down (minority) electrons. In the insets we have blown up the region around the Fermi level.

(LSDA). The self-consistent potentials were calculated on a $20 \times 20 \times 20 \mathrm{k}$-mesh in the Brillouin zone, which corresponds to $256 \mathrm{k}$ points in the irreducible Brillouin zone. The lattice constants were the experimental ones, 0.5756 $\mathrm{nm}$ for $\mathrm{Co}_{2} \mathrm{MnAl}, 0.577 \mathrm{~nm}$ for $\mathrm{Co}_{2} \mathrm{MnGa}, 0.565 \mathrm{~nm}$ for $\mathrm{Co}_{2} \mathrm{MnSi}, 0.574$ for $\mathrm{Co}_{2} \mathrm{MnGe}$ and $0.598 \mathrm{~nm}$ for $\mathrm{Co}_{2} \mathrm{MnSn}$ [5], and were kept constant upon the creation of defects.

\section{Results and discussion}

We have performed calculations for the $\left[\mathrm{Co}_{1-x} \mathrm{Mn}_{x}\right]_{2} \mathrm{MnZ}$ compounds varying the sp atom, $\mathrm{Z}$, which is one of $\mathrm{Al}$, $\mathrm{Ga}, \mathrm{Si}, \mathrm{Ge}$ or Sn. We have taken into account five different values for the concentration $x ; x=0,0.025,0.05,0.1,0.2$. In Fig. 1 we have drawn the total density of states (DOS) for all five families of compounds under study and for three different values of the concentration $x$ : the perfect compounds $(x=0)$ and for two cases with defects, $x=0.1$ and 0.2. In the case of the perfect $\mathrm{Co}_{2} \mathrm{MnSi}$ and $\mathrm{Co}_{2} \mathrm{MnGe}$ compounds there is a real gap in the minority spin band and the Fermi level falls within this gap and these compounds are perfect half-metals. The other three perfect compounds $-\mathrm{Co}_{2} \mathrm{MnAl}, \mathrm{Co}_{2} \mathrm{MnGa}$ and $\mathrm{Co}_{2} \mathrm{MnSn}$ - present in reality a region of tiny minority-spin DOS instead of a real gap, but the spin-polarization at the Fermi level only marginally deviates from the ideal $100 \%$ and these com- 

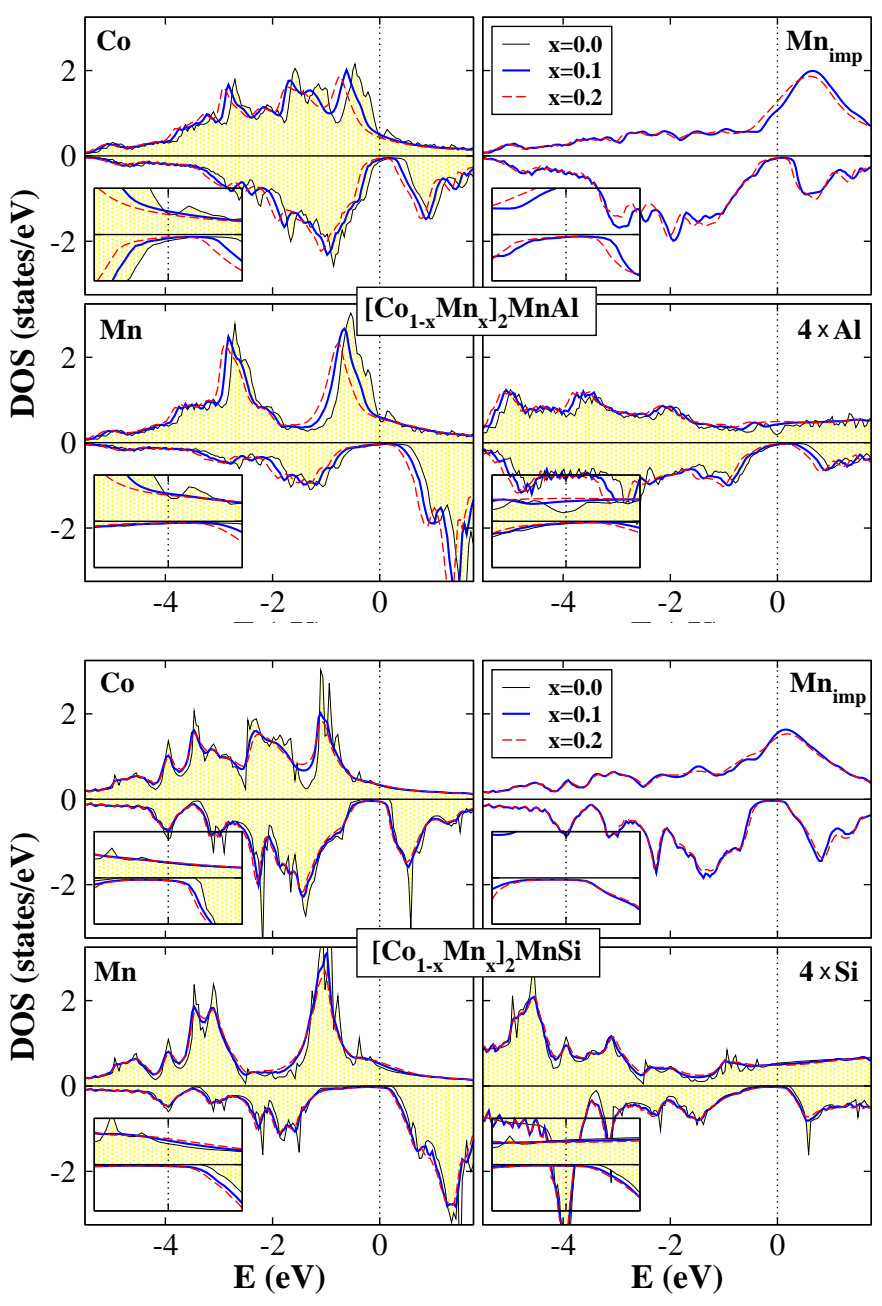

Fig. 2. Atom-resolved DOS as a function of the concentration $x$ for the $\left[\mathrm{Co}_{1-x} \mathrm{Mn}_{x}\right]_{2} \mathrm{MnAl}$ (upper panel) and $\left[\mathrm{Co}_{1-x} \mathrm{Mn}_{x}\right]_{2} \mathrm{MnSi}$ (lower panel) compounds as a function of the concentration $x$. We have used the same notation as in Fig. 1. Note that the atomic DOS's have been scaled to one atom. The DOS for $\mathrm{Al}$ and $\mathrm{Si}$ has been multiplied by a factor of four. Details are similar to Fig. 1.

pounds can be also considered as half-metals. These results agree with previous electronic structure calculations on these compounds $[9,10,29,30,31]$ and are confirmed by the calculated total spin moments as discussed latter in the text. When we create a surplus of Mn atoms which migrate at sites occupied by Co atoms in the perfect alloys, the gap persists and all compounds retain their half-metallic character. This is clearly seen in the insets where we have blown the region around the Fermi level. The most interesting case is the two compounds which presented a real gap, $\mathrm{Co}_{2} \mathrm{MnGe}$ and mainly $\mathrm{Co}_{2} \mathrm{MnSi}$. The creation of $\mathrm{Mn}$ antisites, especially in the Si-case, does not alter the width of the gap and half-metallicity is extremely robust in these alloys with respect to the creation of $\mathrm{Mn}$ antisites. We will explain this behavior latter in the text, when we will discuss the atom-resolved DOS.

In Fig. 2 we have drawn the atom resolved DOS for the $\mathrm{Al}-$ and Si-based compounds presented in Fig. 1. In the perfect compounds, $x=0$, the Co and Mn atoms form a com- mon majority-spin band while the minority occupied states are mainly of Co character. Around the minority-spin bandgap the states are mainly of Co character confirming the results in Ref. [10]. The large exchange splitting between the occupied majority-spin states of Mn atoms and its unoccupied minority states favors the creation of the gap and the appearance of half-metallicity being also responsible for the large spin moments at the Mn sites (see next paragraph). The DOS of the sp atoms is very small with respect to the transition metal atoms and thus we have multiplied it by four to make it visible. Al or Si states around the Fermi level are of $p$ character and they play a central role in the exact position of the Fermi level within the gap (see Ref. [3] for an extended discussion of the role of the sp atoms). When we substitute $\mathrm{Si}$ for $\mathrm{Al}$, we increase the total number of valence electrons in the unit cell by one and this extra electron does not occupy states of the sp atom, which are deep in energy, but rather states of the transition metal atoms [10] provoking small changes in the DOS of the Co and Mn atoms. When we create Mn antisites, the DOS of both Co and Mn atoms at the perfect sites does not significantly change as can be easily observed in Fig. 2 and thus they only marginally affect the half-metallicity. The interesting phenomenon is the behavior of the Mn impurities atoms sitting at the antisites. Now the major weight of the occupied states corresponds to spin-down states and we expect their spin moments to be antiparallel to the ones of the Co and Mn atoms at the perfect sites. Moreover the large exchange splitting of the $\mathrm{Mn}$ atoms ensures a large gap in the spin-down band and the robust character of the half-metallicity. The shape of the DOS of the Mn impurity atoms at the antisites is similar to the one of the $\mathrm{Cr}$ impurity atoms in $\mathrm{Co}_{2} \mathrm{CrAl}$ and $\mathrm{Co}_{2} \mathrm{CrSi}$ [27]. The main advantage of the Mn-based compounds is that when Cr substitutes $\mathrm{Mn}$, the smaller exchange splitting of the $\mathrm{Cr}$ atoms leads to huge narrowing of the band-gap which shrinks to an almost zero value [27]. Thus the Mn compounds have a clear advantage for realistic applications.

Our discussion has been focused mainly to the halfmetallic character of the compounds with the Mn defects. We will now concentrate on the spin moments of the compounds under study to discuss the appearance of the ferrimagnetism in the defected compounds. In Table 1 we have gathered the atom-resolved and total spin moments for the $\left[\mathrm{Co}_{1-x} \mathrm{Mn}_{x}\right]_{2} \mathrm{MnZ}$ alloy where $\mathrm{Z}$ is $\mathrm{Al}$ or its isoelectronic $\mathrm{Ga}$ and for all values of the concentrations $x$ which we have studied, and in Table 2 we present the same information for the $\mathrm{Z}=\mathrm{Si}$, Ge or Sn alloys. We will start our discussion from the calculated total spin moments and how they compare with the ideal values for perfect half-metallicity (values in parenthesis in the tables). These ideal values stem from the Slater-Pauling behavior shown for the full-Heusler alloys [10] which states that the total spin moment in the unit cell in $\mu_{B}$ is the total number of valence electrons in the unit cell, $z_{t}$, minus " 24 " since there are exactly 12 occupied minority-spin states for all half-metallic full-Heuslers. For the perfect compounds this 
Table 1

Atom-resolved spin magnetic moments (in $\mu_{B}$ ) for the $\left[\mathrm{Co}_{1-x} \mathrm{Mn}_{x}\right]_{2} \mathrm{MnZ}$ compounds (moments have been scaled to one atom), where $\mathrm{Z}$ is $\mathrm{Al}$ or Ga. The last column is the total spin moment (Total) in the unit cell calculated as $2 \times\left[(1-x) * m^{C o}+x *\right.$ $\left.m^{M n(i m p)}\right]+m^{M n}+m^{Z}$ and in parenthesis the ideal total spin moment predicted by the Slater-Pauling rule for half-metals (see Ref. [10]). With $\mathrm{Mn}$ (imp) we denote the $\mathrm{Mn}$ atoms sitting at perfect Co sites.

\begin{tabular}{|c|c|c|c|c|}
\hline \multirow[b]{2}{*}{$x$} & \multicolumn{4}{|c|}{$\left[\mathrm{Co}_{1-x} \mathrm{Mn}_{x}\right]_{2} \mathrm{MnAl}$} \\
\hline & Co & $\operatorname{Mn}(\mathrm{imp})$ & $\mathrm{Mn} \quad \mathrm{Al}$ & Total(Ideal) \\
\hline 0 & 0.68 & - & $2.82-0.14$ & $4.04(4.0)$ \\
\hline 0.025 & 0.71 & -2.63 & $2.82-0.14$ & $3.92(3.9)$ \\
\hline 0.05 & 0.73 & -2.59 & $2.82-0.13$ & $3.81(3.8)$ \\
\hline 0.1 & 0.78 & -2.49 & $2.83-0.12$ & $3.61(3.6)$ \\
\hline \multirow[t]{2}{*}{0.2} & 0.84 & -2.23 & $2.85-0.09$ & $3.20(3.2)$ \\
\hline & \multicolumn{4}{|c|}{$\left[\mathrm{Co}_{1-x} \mathrm{Mn}_{x}\right]_{2} \mathrm{MnGa}$} \\
\hline$x$ & Co & $\operatorname{Mn}(\mathrm{imp})$ & $\mathrm{Mn} \quad \mathrm{Ga}$ & Total(Ideal) \\
\hline 0 & 0.65 & - & $2.90-0.10$ & $4.09(4.0)$ \\
\hline 0.025 & 0.68 & -2.76 & $2.90-0.10$ & $4.00(3.9)$ \\
\hline 0.05 & 0.71 & -2.73 & $2.91-0.09$ & $3.89(3.8)$ \\
\hline 0.1 & 0.75 & -2.66 & $2.92-0.08$ & $3.66(3.6)$ \\
\hline 0.2 & 0.82 & -2.42 & $2.94-0.05$ & $3.23(3.2)$ \\
\hline
\end{tabular}

means a total spin moment of $4 \mu_{B}$ for $\mathrm{Co}_{2} \mathrm{MnAl}$ and $\mathrm{Co}_{2} \mathrm{MnGa}$ since they have 28 valence electrons in the unit cell and a spin moment of $5 \mu_{B}$ for the $\mathrm{Co}_{2} \mathrm{MnSi}, \mathrm{Co}_{2} \mathrm{MnGe}$ and $\mathrm{Co}_{2} \mathrm{MnSn}$ compounds which have one electron more. The calculated total spin moments are exactly $5 \mu_{B}$ for the perfect $\mathrm{Co}_{2} \mathrm{MnSi}$ and $\mathrm{Co}_{2} \mathrm{MnGe}$ alloys and only slightly deviate from the ideal values for the other three compounds confirming the discussion on the total DOS. When we induce the Mn impurities at the antisites, we have to take the average value of the valence electrons calculated as $2 \times\left[(1-x) * z^{C o}+x * z^{M n(i m p)}\right]+z^{M n}+z^{\text {sp atom }}$ where $z$ is the number of valence electrons of the corresponding chemical element. Mn is lighter than Co and thus as we increase the concentration in $\mathrm{Mn}$ defects, the total spin moment should decrease. In the case of the $\left[\mathrm{Co}_{1-x} \mathrm{Mn}_{x}\right]_{2} \mathrm{Mn}-\mathrm{Si}$ and -Ge compounds the ideal half-metallicity is preserved irrespectively of the concentration of the Mn impurities. The $\mathrm{Al}$ and Sn compounds are almost half-metallic while slightly larger deviations are observed for the Ga compound. Overall we could safely state that half-metallicity is preserved upon the creation of $\mathrm{Mn}$ antisites.

The spin moment of Mn atoms at the perfect sites remains practically constant for all five families of compounds under study, when the concentration in Mn defects is increased, since their environment does not significantly change; each $\mathrm{Mn}$ atom has eight Co atoms as first neighbors in the perfect alloy and for the values of the concentration under study here their environment remains of mainly Co character. Co atoms in the case of $\mathrm{Si}, \mathrm{Ge}$ and Sn compounds retain a practically constant moment while in $\mathrm{Al}$ and $\mathrm{Ga}$ compounds it slightly increases. This
Table 2

Similar to Table 1 for the $\left[\mathrm{Co}_{1-x} \mathrm{Mn}_{x}\right]_{2} \mathrm{MnZ}$ compounds with $\mathrm{Z}=$ $\mathrm{Si}, \mathrm{Ge}$ or Sn.

\begin{tabular}{|c|c|c|c|c|}
\hline \multirow[b]{2}{*}{$x$} & \multicolumn{4}{|c|}{$\left[\mathrm{Co}_{1-x} \mathrm{Mn}_{x}\right]_{2} \mathrm{MnSi}$} \\
\hline & Co & $\mathrm{Mn}(\mathrm{imp})$ & $\mathrm{Mn} \quad \mathrm{Si}$ & Total(Ideal) \\
\hline 0.0 & 0.98 & - & $3.13-0.09$ & $5.00(5.0)$ \\
\hline 0.025 & 0.99 & -1.01 & $3.11-0.09$ & $4.90(4.9)$ \\
\hline 0.05 & 0.99 & -0.95 & $3.09-0.08$ & $4.80(4.8)$ \\
\hline 0.1 & 0.99 & -0.84 & $3.06-0.07$ & $4.60(4.6)$ \\
\hline \multirow[t]{2}{*}{0.2} & 0.97 & -0.70 & $2.99-0.05$ & $4.20(4.2)$ \\
\hline & \multicolumn{4}{|c|}{$\left[\mathrm{Co}_{1-x} \mathrm{Mn}_{x}\right]_{2} \mathrm{MnGe}$} \\
\hline$x$ & Co & $\operatorname{Mn}(\mathrm{imp})$ & $\mathrm{Mn} \quad \mathrm{Ge}$ & Total(Ideal) \\
\hline 0 & 0.93 & - & $3.20-0.06$ & $5.00(5.0)$ \\
\hline 0.025 & 0.94 & -1.72 & $3.21-0.06$ & $4.90(4.9)$ \\
\hline 0.05 & 0.95 & -1.63 & $3.20-0.05$ & $4.80(4.8)$ \\
\hline 0.1 & 0.97 & -1.45 & $3.19-0.04$ & $4.60(4.6)$ \\
\hline \multirow[t]{2}{*}{0.2} & 0.96 & -1.18 & $3.15-0.02$ & $4.20(4.2)$ \\
\hline & \multicolumn{4}{|c|}{$\left[\mathrm{Co}_{1-x} \mathrm{Mn}_{x}\right]_{2} \mathrm{MnSn}$} \\
\hline$x$ & Co & $\mathrm{Mn}(\mathrm{imp})$ & $\mathrm{Mn} \quad \mathrm{Sn}$ & Total(Ideal) \\
\hline 0 & 0.89 & - & $3.32-0.08$ & $5.02(5.0)$ \\
\hline 0.025 & 0.91 & -2.44 & $3.34-0.07$ & $4.91(4.9)$ \\
\hline 0.05 & 0.93 & -2.34 & $3.35-0.06$ & $4.81(4.8)$ \\
\hline 0.1 & 0.96 & -2.14 & $3.35-0.05$ & $4.60(4.6)$ \\
\hline 0.2 & 0.98 & -1.73 & $3.35-0.03$ & $4.20(4.2)$ \\
\hline
\end{tabular}

implies that in the case of $\mathrm{Si}$, Ge and Sn-based compounds Co atoms show a more atomic like behavior and they are not strongly affected by their environment and thus their spin moment is around $1 \mu_{B}$. In the case of $\mathrm{Al}$ and $\mathrm{Ga}$ compounds Co atoms are more affected by their environment and for this reason their spin moment in the perfect compounds is smaller. As we increase the concentration of Mn defects in these two alloys, also the spin moment of the Co atoms increases reaching a value of $\sim 0.8 \mu_{B}$. Since the spin moments of both Co and Mn atoms at the perfect sites do not present drastic changes, the only way to achieve the half-metallicity for the compounds (and thus to decrease their total spin moment) is that the $\mathrm{Mn} \mathrm{impu-}$ rity atoms at the antisites have spin moments antiparallel to the other transition metal atoms. This is actually the case as can be seen in Tables 1 and 2. Mn impurity atoms have an important negative spin moment especially in the case of the $\mathrm{Al}$ and $\mathrm{Ga}$ compounds where it attends 2.6-2.8 $\mu_{B}$. It slightly drops as we increase the concentration in $\mathrm{Mn}$ defects but this is expected since we increase also the hybridization between Mn impurity atoms; Mn atoms contrary to Co atoms have more expanded $d$-like wavefunctions and hybridize stronger between them.

Of particular interest is the case of $\mathrm{Si}, \mathrm{Ge}$ and $\mathrm{Sn}$ compounds, where the spin moment of the Mn impurity atoms shows large variations between the three families of alloys. As we pass from Si to Ge and then to Sn-based al- 
loy, its spin moment for $x=0.025$ increases by $-0.72 \mu_{B}$. Although the spin moment of the Mn impurity atom changes significantly, its contribution to the total spin moment is small. A large increase of the absolute value of the spin moment of the Mn impurities, as the one observed here, means a small increase of its negative contribution to the total spin moment which has to balance the larger positive contribution of the spin moment of the Mn atoms at the perfect sites observed for the compound with the heavier sp element. For the Ge- and Sn-compounds the DOS has a similar shape to the one for the Si-based alloys (and thus they are not shown here), but the Fermi level is lower in energy with respect the Si-based compound. Since the majority DOS takes large values at the region of the minority gap, this small shift of the Fermi level provokes a considerable increase of the negative spin moment of the Mn impurity atoms. Thus the Mn-doped alloys are half-metallic ferrimagnets and their total spin moment is considerable smaller than the perfect half-metallic ferromagnetic parent compounds. A similar phenomenon has been also predicted when creating $\mathrm{Cr}$ antisites in the zinc-blende CrAs intermetallic alloy [26] where $\mathrm{Cr}$ impurities spin moments couple antiferromagnetically to the $\mathrm{Cr}$ atoms at the ideal sites in the zinc-blende structure. Similarly $\mathrm{Cr}$ antisites in $\mathrm{Co}_{2} \mathrm{CrAl}$ and $\mathrm{Co}_{2} \mathrm{CrSi}$ compounds lead to a similar phenomenon [27]. The importance of the present results stem from the more intense research on the compounds containing $\mathrm{Co}$ and $\mathrm{Mn}$ and from their more robust half-metallic character, since in the case of $\mathrm{Cr}$ compounds the gap almost vanishes upon the creation of $\mathrm{Cr}$ defects. Here we have to mention that if also Co atoms migrate to Mn sites (case of atomic swaps) the half-metallity is lost, as it was shown by Picozzi et al. [35], due to the energy position of the Co states which have migrated at Co sites.

\section{Summary and conclusions}

We have studied the effect of defects-driven appearance of half-metallic ferrimagnetism in the case of the $\mathrm{Co}_{2} \mathrm{MnZ}$ Heusler alloys, where Z stands for Al, Ga, Si, Ge or Sn. More precisely, based on first-principles calculations we have shown that when we create $\mathrm{Mn}$ antisites at the Co sites, these impurity $\mathrm{Mn}$ atoms couple antiferromagnetically with the Co and the Mn atoms at the perfect sites while keeping the half-metallic character of the parent compounds. Half-metallicity in these compounds is a robust property since the large exchange splitting of the Mn impurity atoms ensures that the width of the gap in the minority spin band only marginally is affected. Thus we have shown an alternative way to create robust half-metallic ferrimagnets, which are crucial for magnetoelectronic applications, based in the introduction of defects in half-metallic ferromagnetic Heusler alloys which are widely studied. Especially the case of $\left[\mathrm{Co}_{1-x} \mathrm{Mn}_{x}\right]_{2} \mathrm{MnSi}$ alloys is of particular interest since $\mathrm{Mn}_{3} \mathrm{Si}$ is known to crystallize in the Heusler $L 2_{1}$ lattice structure of $\mathrm{Co}_{2} \mathrm{MnSi}$. We expect these results to stimulate further interest in the theoretical and experimental research in the field of spintronics.

\section{References}

[1] I. Žutić, J. Fabian, S. Das Sarma, Rev. Mod. Phys. 76 (2004) 323.

[2] Half-metallic alloys: fundamentals and applications, Eds.: I. Galanakis and P.H. Dederichs, Lecture notes in Physics vol. 676 (Berlin Heidelberg: Springer 2005).

[3] I. Galanakis, Ph. Mavropoulos, P.H. Dederichs, J. Phys. D: Appl. Phys. 39 (2006) 765.

[4] R.A. de Groot. F.M. Mueller, P.G. van Engen, K.H.J. Buschow, Phys. Rev. Lett. 50 (1983) 2024.

[5] P. J. Webster and K. R. A. Ziebeck, in Alloys and Compounds of d-Elements with Main Group Elements. Part 2., edited by H. R. J. Wijn, Landolt-Boörnstein, New Series, Group III, Vol. 19,Pt.c (Springer-Verlag, Berlin 1988), pp. 75-184.

[6] I. Galanakis, P.H. Dederichs, N. Papanikolaou, Phys. Rev. B 66 (2002) 134428.

[7] P.J. Webster, J. Phys. Chem. Solids 32 (1971) 1221.

[8] S. Ishida, S. Fujii, S. Kashiwagi, S. Asano, J. Phys. Soc. Jpn. 64 (1995) 2152; S. Fujii, S. Sugimura, S. Ishida, S. Asano, J. Phys.: Condens. Matter 2 (1990) 8583.

[9] S. Picozzi, A. Continenza, A.J. Freeman, Phys. Rev. B (2002) 094421.

[10] I. Galanakis, P.H. Dederichs, N. Papanikolaou, Phys. Rev. B 66 (2002) 174429

[11] A. Bergmann, J. Grabis, B.P. Toperverg, V. Leiner, M. Wolff, H. Zabel, K. Westerholt, Phys. Rev. B 72 (2005) 214403; J. Grabis, A. Bergmann, A. Nefedov, K. Westerholt, H. Zabel, Phys. Rev. B 72 (2005) 024437; idem, Phys. Rev. B 72 (2005) 024438.

[12] A. Rata, H. Braak, D.E. Bürgler, S. Cramm, and C.M. Schneider, Eur. Phys. J. B 52 (2006) 445; M. Kallmayer H. Schneider, G. Jakob, H.J. Elmers, K. Kroth, H.C. Kandpal, U. Stumm, S. Cramm, Appl. Phys. Lett. 88 (2006) 072506; S.V. Karthik, A. Rajanikanth, Y.K. Takahashi, T. Okhubo, K. Hono, Appl. Phys. Lett. 89 (2006) 052505; G.H. Fecher, H.C. Kandpal, S. Würmehl, J. Morais, H.-J. Lin, H.-J. Elmers, G. Schönhense, C. Felser, J. Phys.: Condens. Matter 17 (2005) 7237; R.Y. Umetsu, K. Kobayashi, A. Fujita, K. Oikawa, R. Kainuma, K. Ishida, N. Endo, K. Fukamichi, A. Sakuma, Phys. Rev. B 72 (2005) 214412; K. Kobayashi, R.Y. Umetsu, R. Kainuma, K. Ishida, T. Oyamada, A. Fujita, K. Fukamichi, Appl. Phys. Lett. 85 (2004) 4684; H.J. Elmers, G.H. Fecher, D. Valdaitsev, S.A. Nepijko, A, Gloskovskii, G. Jakob, G. Schönhense, S. Wurmehl, T. Block, C. Felser, P.-C. Hsu, W.-L. Tsai, S. Cramm, Phys. Rev. B 67 (2003) 104412.

[13] T. Marukame, T. Ishikawa, K.-I. Matsuda, T. Uemura, M. Yamamoto, Appl. Phys. Lett. 88 (2006) 262503; T. Marukame, T. Kasahara, K. Matsuda, T. Uemura T M. Yamamoto, Jpn. J. Appl. Phys. 44 (2005) L521.

[14] R. Kelekar and B.M. Klemens, Appl. Phys. Lett. 86 (2005) 232501; R. Kelekar, H. Ohldag, B.M. Clemens, Phys. Rev. B 75 (2007) 014429.

[15] S. Kämmerer, A. Thomas, A. Hütten, G. Reiss, Appl. Phys. Lett. 85 (2004) 79; J. Schmalhorst, S. Kämmerer, M. Sacher, G. Reiss, A. Hütten, A. Scholl, Phys. Rev. B 70 (2004) 024426.

[16] Y. Sakuraba, M. Hattori, M. Oogane, Y. Ando, H. Kato, A. Sakuma, T. Miyazaki, H. Kubota, Appl. Phys. Lett. 88 (2006) 192508; Y. Sakuraba, J. Nakata, M. Oogane, Y. Ando, H. Kato, A. Sakuma, T. Miyazaki, H. Kubota, Appl. Phys. Lett. 88 (2006)022503; Y. Sakuraba, T. Miyakoshi, M. Oogane, Y. Ando, A. Sakuma, T. Miyazaki, H. Kubota, Appl. Phys. Lett. 89 (2006) 052508; X.Y. Dong, C. Adelmann, J.Q. Xie, C.J. Palmström, X. 
Lou, J. Strand, P.A. Crowell, J.-P. Barnes, A.K. Petford-Long, Appl. Phys. Lett. 86 (2005) 102107.

[17] W.H. Wang, M. Przybylski, W. Kuch, L.I. Chelaru, J. Wang, Y.F. Lu, J. Barthel, H.L. Meyerheim, J. Kirschner, Phys. Rev. B 71 (2005) 144416; S. Wurmehl, G.H. Fecher, H.C. Kandpal, V. Ksenofontov, C. Felser, H.-J. Lin, J. Morais, Phys. Rev. B 72 (2005) 184434; R.Y. Umetsu, K.Kobayashi, R.Kainuma, A. Fujita, K. Fukamichi, K.Ishida, A. Sakuma, Appl. Phys. Lett. 85 (2004) 2011; L. Ritchie, G. Xiao, Y. Ji, T.Y. Chen, C.L. Chien, M. Zhang, J. Chen, Z. Liu, G. Wu, X.X. Zhang, Phys. Rev. B 68 (2003) 104430.

[18] K. Yakushiji, K. Saito, S. Mitani, K. Takanashi, Y.K. Takahashi, K. Hono, Appl. Phys. Lett. 88 (2006) 222504; M. Hashimoto, J. Herfort, H.-P. Schönherr, K.H. Ploog, Appl. Phys. Lett. 87 (2005) 102506.

[19] N.-N. Liu, A. Thomas, G. Reiss, A. Hütten, Appl. Phys. Lett. 89 (2006) 162506; Z. Gercsi, A. Rajanikanth, Y.K. Takahashi, K. Hono, M. Kikuchi, N. Tezuka, K. Inomata, Appl. Phys. Lett. 89 (2006) 082512.

[20] N. Tezuka, N. Ikeda, A. Miyazaki, S. Sugimoto, M. Kikuchi, K. Inomata, Appl. Phys. Lett. 89 (2006) 112514; S. Okamura, A. Miyazaki, S. Sugimoto, N. Tezuka, K. Inomata, Appl. Phys. Lett. 86(2005) 232503.

[21] E. Şaşığlu, L. M. Sandratskii, P. Bruno, and I. Galanakis, Phys. Rev. B 72, 184415 (2005).

[22] H. van Leuken and R.A. de Groot, Phys. Rev. Lett. 74 (1995) 1171; S. Wurmehl, H.C. Kandpal, G.H. Fecher, C. Felser, J. Phys.: Condens. Matter 18 (2006) 6171.

[23] R.A. de Groot, A.M. van der Kraan, K.H.J. Buschow, J. Magn. Magn. Mater. 61 (1986) 330.

[24] K. Özdog̃an, I. Galanakis, E. Şaşıoglu, B. Aktaş, J. Phys.: Condens. Matter 18 (2006) 2905; E. Şaşıõlu, L.M. Sandratskii, P. Bruno, J. Phys.: Condens. Matter 17 (2005) 995.

[25] H. Akai and M. Ogura, Phys. Rev. Lett. 97 (2006) 026401.

[26] I. Galanakis, K. Özdog̃an, E. Şaşıog̃lu, B. Aktaş, Phys. Rev. B 74 (2006) 140408(R).

[27] K. Özdog̃an, I. Galanakis, E. Şaşıõlu, B. Aktaş, Phys. Stat. Sol. (RRL) 1 (2007) R95.

[28] X.-Q. Chen, R. Podloucky, P. Rogl, J. Appl. Phys. 100 (2006) 113901.

[29] M. Sargolzaei, M. Richter, K. Koepernik, I. Opahle, H. Eschrig, I. Chaplygin, Phys. Rev. B 74 (2006) 224410.

[30] K. Özdog̃an, B. Aktaş, I. Galanakis, E. Şaşıỡlu, J. Appl. Phys. in press [preprint cond-mat/0612194

[31] I. Galanakis, K. Ozdog̃an, B. Aktaş, E. Şaşıỡlu, Appl. Phys. Lett. 89 (2006) 042502; K. Özdog̃an, E. Şaşığlu, B. Aktaş, I. Galanakis, Phys. Rev. B 74 (2006) 172412; I. Galanakis, J. Phys.: Condens. Matter 16 (2004) 3089.

[32] V.N. Antonov, H.A. Dürr, Yu. Kucherenko, L.V. Bekenov, A.N. Yaresko, Phys. Rev B 72 (2005) 054441; Y. Miura, K. Nagao, M. Shirai, Phys. Rev B 69 (2004) 144413; B. Balke, G.H. Fecher, H.C. Kandpal, C. Felser, K. Kobayashi, E. Ikenaga, J.-J. Kim, S. Ueda, Phys. Rev. B 74 (2006) 104405.

[33] C. Pfleiderer, J. Boeuf, H.v. Löhneysen, Phys. Rev. B 65 (2002) 172404.

[34] J.W. Cable, N. Wakabayashi, P. Radhakrishna, Phys. Rev. B 48 (1993) 6159.

[35] S. Picozzi, A. Continenza, A.J. Freeman, Phys. Rev. B 69 (2004) 094423.

[36] K. Koepernik and H. Eschrig, Phys. Rev. B 59 (1999) 3174; K. Koepernik, B. Velicky, R. Hayn, H. Eschrig, Phys. Rev. B 58 (1998) 6944. 